\title{
PERFORMANCE IMPROVEMENT OF IDMA SCHEME USING CHAOTIC MAP INTERLEAVERS FOR FUTURE RADIO COMMUNICATION
}

\author{
Aasheesh Shukla ${ }^{1}$ and Vinay Kumar Deolia ${ }^{2}$ \\ Department of Electronics and Communications Engineering, Institute of Engineering and Technology, GLA University, India
}

\begin{abstract}
In this paper, chaos based interleavers are proposed for the performance improvement of Interleave Division Multiple Access (IDMA henceforth) for future radio communication (FRC) requirements. 'IDMA' can be mean as the modified case of direct sequence code division multiple access (DS-CDMA) with the same spreading sequences and user specific interleavers for distinguishing the users in multi-user environment. In IDMA systems, the roles of interleavers are pre-eminent and an efficient interleaver contributes in optimizing the system performance. Random interleaver is the popular and basic taxonomy in IDMA. The performance of chaos based interleavers is compared to random interleaver. Simulation results authenticate the performance of chaos based IDMA. Further the proposed chaotic map-interleavers have the less computational complexity and efficient in bandwidth compared to the existing prevailing interleaver algorithms in the domain. The IDMA system model uses a BPSK modulation and repetition coder with a code rate of $1 / 2$. The system is simulated in MATLAB and results show the BER superiority of chaotic interleaver based IDMA without the need of extra storage resources and less computational complexity.
\end{abstract}

Keywords:

IDMA, Chaos, Tent Map, Logistic Interleaver, CDMA

\section{INTRODUCTION}

IDMA is one of the popular spread spectrum multiple access scheme which can be seen as a special case direct sequence code division multiple access (CDMA for short hence forth). In IDMA interleavers are used for user separation rather than by spreading sequences like in CDMA. Interleavers are the crucial part of wireless communication, specifically over noisy channel. Chip interleaving was first introduced to combat the effect of burst impulsive noise disturbance. The interleaver scrambles the ordering of bits in sequences and hence converts the bursty channel into random error channel [1].

CDMA was widely used in third generation mobile communication system but the performance of this technique has been limited due to the mutual interference among multiple users (MAI) and inters symbol interference (ISI). Although several researches are given for the cancellation of MAI such as successive interference cancellation (SIC) and parallel interference cancellation(PIC), but these challenges in CDMA restrict its role in future communication. In order to enhance the spectrum efficiency, Ping et al. proposed a new multiple access scheme i.e. Interleave division multiple access (IDMA) [2]. However the performance of IDMA is improved, because the entire bandwidth expansion is devoted to the coding and interleavers are dedicated to distinguish the users. Hence IDMA inherits many advantages of CDMA and also allows a simple iterative Multi User Detection (MUD) strategy [3]. With many benefits, IDMA offers some challenges too. Some of them can be listed as:

- Bandwidth requirement: Interleaving sequence is also sent along with information bits and hence it increases the bandwidth requirement.

- Computational Complexity: Each user is assigned a different interleaver which enhances the system complexity.

- Storage resources requirement: Interleaving algorithm is needed to store at the side of transmitter as well as at receiver which may become a major challenge, especially when number of user is large.

To conquer above limitations, researchers have suggested different design and algorithms of interleavers. There are many popular interleaver designs available in the literature such as Orthogonal Interleaver (OI), Pseudo-random interleaver, Tree based Interleaver (TBI), Helical Interleaver, Nested Interleaver, Shifting Interleaver, Deterministic Interleavers and many more [4][5][6]. Based on the work carried in the research, it can be concluded that the choice of optimum interleaver may have a great impact on the performance of IDMA system. Ramsey reported the basic interleaver design in 1970 .

The paper focus on the random interleaving algorithm to minimize the delay and storage resources requirement. However, the randomly generated interleaving pattern also has some limitations, such as large storage requirement and high complexity. Pupeza et al. [7] proposed the generation of Nested interleaver (NI), which choose the first interleaver sequence from pseudo-random interleaver and rest interleavers are generated by re-ordering procedure with the limitation that, it consumes large memory and bandwidth, although, the bit error rate (BER) performance was better than random interleaver. Pupeza et al. also presented Orthogonal Interleaver (OI), which guarantee the users to be orthogonal and hence diminish the effect of multiple access interference [7][8]. However the performance of OI is limited due the requirement of large number of orthogonal sequences for simultaneous users. Zhang proposed a shifting interleaver for IDMA scheme and cyclically shifting interleaver. Both the interleavers were not suitable for MUD receiver. Tseng et al. suggested a novel design of deterministic interleaver which was firstly used in turbo codes. The design of this interleaver was less complex and more bandwidth efficient with the only limitation that the use of look up table, the need of the table increases the memory requirement at the transmitter [8]. Shukla et al. also suggested a new Tree Based Interleaver with the scope of further improvement in memory requirement. Many other algorithms of interleaver design also have been proposed by researchers but the journey towards the search of optimum interleaver is still on for making IDMA suitable for future communication [9]. 


\subsection{MOTIVATION}

Recently the use of chaos in spread spectrum communication drawn the attention of researchers and this is found that the use of chaos into spread spectrum communication systems offers many advantages as well as provides several opportunities for the improvement is system performance. Chaotic systems are a basically non-dynamical system which highly depends upon on initial conditions and consequently, may help in the generation of uncorrelated, random-like, yet deterministic and reproducible signals or sequences [10]. Manoharan et al. presented the analysis of chaos based codes and assured the better performance of chaotic codes in comparison of $p-n$ code. Chaotic sequences shows good decorrelation properties and hence reduce multiple access interference (MAI) [11]-[14] Further different chaotic maps are used to generate sequences and so interleavers for IDMA system. The popular chaotic maps are Logistic map, Tent map, Bernoulli's map, Baker Map, Henon map etc. The current work proposes a solution for the search of an efficient interleaver. In this article, the logistic and tent map based interleavers are analyzed in IDMA scheme and assures the better performance.

The content of the paper is organized as follows. Section 2 presents the IDMA system description. Section 3 defines chaos based algorithms for interleaver design. Section 4, develops the performance analysis of proposed interleaver with IDMA schemes and section 5 discusses the conclusions drawn on the basis of BER performance analysis and computational complexity.

\section{SYSTEM DESCRIPTION}

Chip level interleaving was popularly used to mitigate the effect of burst impulsive noise disturbances of the channel. It also motivates that the users should be separated by chip level interleaver rather than by spreading codes as in conventional CDMA system. The system is popularly named as Interleave division multiple accesses. The block diagram of Interleave Division Multiple Access (IDMA) system with $K$ users is presented in Fig. 1. At the transmitter, the information bits $b_{i, k}$ are encoded by the encoder of rate $R$. The spreader code is simply a repetition code of rate $R_{r}$. The resulting code bits $c_{j, k}, j \in\left[1, \ldots, N_{b}\right]$ are interleaved by user specific interleaver $\prod_{k}$ to get $c_{m, n, k}^{\prime}$ i.e.

$c_{k}=\left[c_{k 1}, c_{k 2}, \ldots, c_{k i}\right]$, where $j$ is the chip length. Using the effective channel the received signal can be represented as $y_{n}$. Here the transmitted symbol can be written as $x_{n} \triangleq\left[x_{n 1}, \ldots ., x_{n K}\right]^{T}$ and noise vector may be $\zeta_{n+l} \triangleq\left[\zeta_{n}+l, \ldots ., \zeta_{n}+(l+1)\right]^{T}$. In receiver section, the received signal can be described as:

$$
y_{n}=H_{n} x_{n}+\zeta_{n}
$$

The partition of effective channel matrix can be further defined as

$$
H_{n}=\left[\left[h_{n-\ell, 1}, \ldots, h_{n-\ell, K}\right], \ldots,\left[h_{n, 1}, \ldots, h_{n, K}\right], \ldots,\left[h_{n+\ell, 1}, \ldots, h_{n+\ell, K}\right]\right.
$$

The input to the receiver can be written as:

$$
y_{n}=\sum_{K=1}^{K} \sum_{\ell=-\ell}^{\ell} h_{n-\ell, K} x_{n-\ell, K}+\zeta_{n}
$$

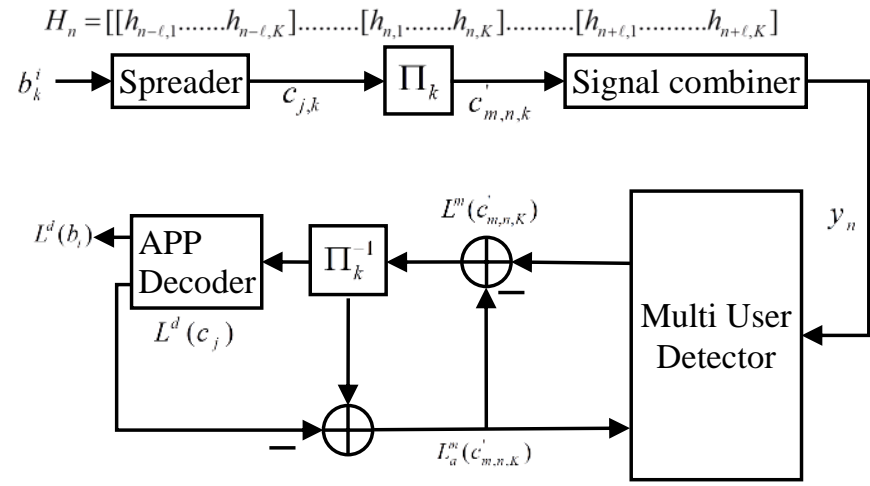

Fig.1. IDMA transmitter and receiver for $k^{\text {th }}$ user

The receiver for IDMA system of $K$ simultaneously transmitting users are comprised of multiuser detector (MUD) and $K$ posteriori probability (APP) decoders in connection with user specific interleavers. The APP decoders and interleavers exchange the soft information and calculate the log likelihood ratios (LLRs) [4]. These LLRs should be independent from the users and hence called as extrinsic LLRs. The goal of the multi user detector is to compute the a posteriori LLRs, which can be represented as,

$$
L^{m}\left(c_{m, n, k}^{\prime}\right)=\ln \frac{P\left(c_{m, n, k}^{\prime}=+1 / y_{n}\right)}{P\left(c_{m, n, k}^{\prime}=-1 / y_{n}\right)}
$$

Now the extrinsic LLRs can be calculated accordingly i.e. $L_{c}^{m}\left(c_{m, n, k}^{\prime}\right)=L^{m}\left(c_{m, n, k}^{\prime}\right)-L_{a}^{m}\left(c_{m, n, k}^{\prime}\right)$ are sent to the decoder after deinterleaving operation i.e. $\Pi_{k}^{-1}$. Now by using total probability theorem the extrinsic LLR can be written as,

$$
L_{c}^{m}\left(c_{m, n, k}^{\prime}\right)=\ln \frac{\sum_{C_{m, n, k+1}} P\left\{y_{n} \mid x_{n, k}=x\right\} \prod_{m \neq m} P\left\{c_{m, n, k}^{\prime}=d_{m}\right\}}{\sum_{C_{m, n, k=1}^{\prime}} P\left\{y_{n} \mid x_{n, k}=x\right\} \prod_{m \neq m} P\left\{c_{m, n, k}^{\prime}=d_{m}\right\}}
$$

Here, $d_{m^{\prime}}=\{+1,-1\}$ denotes the $m^{\text {th }}$ bit of symbol $x$. Each of decoder receives the de-interleaved extrinsic LLRs and channel LLRs $L_{c}^{d}$. Now with some constraints the decoder computed a posteriori LLRs $L^{d}$.

To get extrinsic LLRs, the channel LLRs are subtracted from posteriori LLRs i.e. $L_{e}^{d}=L^{d}-L_{c}^{d}$. After some iteration, the decoder calculates a posteriori LLRs about the information bits $L^{d}\left(b_{i}\right)$. Taking the proper sign, it gives an estimate of information bits.

Table.1. Parameters and description

\begin{tabular}{|c|c|}
\hline Symbols & Description \\
\hline$b_{i, k}$ & Information bit for user $k$ \\
\hline$c_{j, k}$ & Coded bits \\
\hline$c_{m, n, k}$ & Interleaver output \\
\hline$x_{n}$ & Transmitted sequence \\
\hline$\zeta_{n}$ & Noise vector \\
\hline$L^{m}\left(c_{m, n, k}^{\prime}\right)$ & Extrinsic LLR \\
\hline$L_{e}^{d}$ & Channel LLR \\
\hline
\end{tabular}




\section{INTERLEAVER DESIGN}

It is noted that any system may be chaotic if it follows certain properties, such as the system should be deterministic, noise like random in nature and its sensitive dependence on initial conditions so that different signals could be generated. Some of the popular chaotic maps are shown in Table.2, such as Logistic map, Tent map, Bernoulli's map, Baker Map, Henon map etc. Here the algorithm of interleaver design is proposed on the basis of logistic map and tent map. The performance of chaotic IDMA is evaluated and compared using conventional and popular interleavers.

\subsection{LOGISTIC MAP INTERLEAVER}

Here, first the modified logistic map [15] is used for the generation of interleaver sequence. The NLM is described as,

$$
F_{m+1}(x)=\mu x_{n}\left(1-\frac{x_{n}}{N}\right)
$$

where, $x_{n} \in[0, N]$ and $N$ is the interleaver length. Further the above system Eq.(5) exhibits chaotic behavior for positive Lyapunov exponents and for bifurcation parameter, $\mu \geq 3.58$. In this paper the value of $\mu=4$ is taken for the purpose of simulation. To design the interleaver, first the states are calculated for some predefined initial value. All of these states belong to the set of real values between 0 and $N$. Now either by maximizing or ceiling function, all the states transit to integer values and remove the decimal values. Finally, the first interleaver sequence can be obtained after eliminating the redundant bits. Further, to get second interleaver sequence, the initial value of state is modified by adding a foot step to the previous state value i.e. $x_{0}^{2}=x_{0}^{1}+\zeta$.

However all of the above mentioned steps are repeated to get the second interleaver and so on [13-15].

Table.2. Chaotic Maps

\begin{tabular}{|c|c|}
\hline Chaotic Map & Equation \\
\hline Logistic Map & $F_{m+1}(x)=\mu x_{n}\left(1-\frac{x_{n}}{N}\right)$ \\
\hline Tent Map & $F_{m}(x)=\left\{\begin{array}{ccc}m x_{n} & \text { if } & 0 \leq x \leq 0.5 \\
m\left(1-x_{n}\right) & \text { if } & 0.5 \leq x \leq 1\end{array}\right.$ \\
\hline Baker Map & $F(x, y)=\left\{\begin{array}{cc}2 x, \frac{y}{2} & 0<x<0.5 \\
(2 x-1),(y+0.5) & 0.5<x<1\end{array}\right.$ \\
\hline Henon Map & $\begin{array}{l}X_{n+1}=1-a X_{n}^{2}+Y_{n} \\
Y_{n+1}=b X_{n}\end{array}$ \\
\hline
\end{tabular}

\subsection{TENT MAP INTERLEAVER}

Some properties of Tent map are also very encouraging and make this map suitable for the generation of random sequences and consequently interleaving sequences too. Tent map satisfies all above-said conditions required for a map to be chaotic. In the parameterized form, tent map, can be described piecewise by,

$$
F_{m}(x)=\left\{\begin{array}{ccc}
m x_{n} & \text { if } & 0 \leq x \leq 0.5 \\
m\left(1-x_{n}\right) & \text { if } & 0.5 \leq x \leq 1
\end{array}\right.
$$

where, $x_{0}$ is the initial state and $x \in[0,1]$ and parameter ' $m$ ' is known as bifurcation parameter. The Lyapunov exponent (L.E) can also be used to find that either system is chaotic or not. The value of L.E for the particular orbit of function $f(x)$ can be written as [6],

$$
\lambda=\lim _{n \rightarrow \infty}\left\{\frac{1}{n} \sum_{j=0}^{n-1} \ln \left|f^{\prime}(x)\right|\right\}
$$

Now, if $\lambda$ is positive then the system exhibit the chaos in behavior. For example, the above said system Eq.(6) could be analyzed to find the value of lyapunov exponents. The derivative of tent map equations can be further written as,

$$
F^{\prime}(x)=\left\{\begin{array}{c}
m \text { if } 0 \leq x \leq 0.5 \\
-m \text { if } 0.5 \leq x \leq 1
\end{array}\right.
$$

Put the outcome of Eq.(8) in the Eq.(7) to get Lyapunov constant such as,

$$
\lambda=\lim _{n \rightarrow \infty}\left\{\frac{1}{n} \sum_{j=0}^{n-1} \ln | \pm m|\right\}
$$

Further L.E can be simplified to,

$$
\lambda=\lim _{n \rightarrow \infty}\left\{\frac{1}{n} \ln |m|^{n}\right\}=\ln |m| .
$$

Thus, $\lambda$ may be positive for $|m| \geq 1$ and hence the system (TENT map) may be chaotic. Hence Tent map sequences can be used for the generation of interleavers. The flow diagram to generate first interleaver is shown in Fig.2.

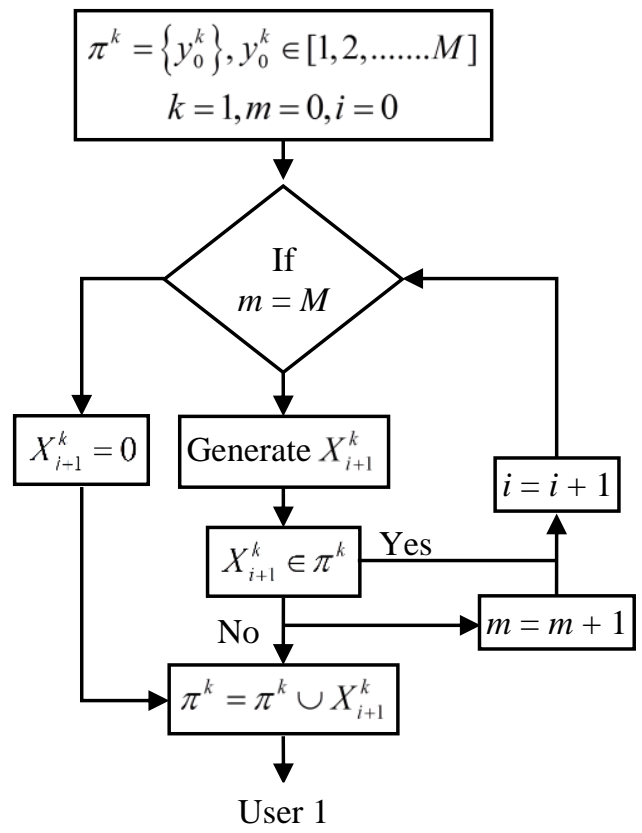

Fig.2. Flow diagram for the generation of first interleaver using Tent map

Here the states of tent map are transit to integer value and redundant bits are removed to generate a distinguished interleaver 
sequence. For the second user, the initial value or first value is modified by foot step $\xi$. Due to change in initial value and chaotic behavior of Tent map, the entirely different interleaver sequence is generated. The process can be repeated to get desired number of interleaver.

\subsubsection{Algorithm:}

$m=2$ (Bifurcation parameter),

$N=$ interleaver length,

$k=$ no. of users.

$X_{j}^{k}=k^{\text {th }}$ user $: 0<X_{j}^{k}<N$,

$\xi=$ Foot step

$F_{0}^{k}=\left\lceil X_{0}^{k}\right\rceil:$ First element $\left(\Pi^{k} \equiv F_{0}^{k}\right)$,

$j=0, n=0$

If $n<N$

$$
\begin{aligned}
& \text { Calculate } X_{j+1}^{k} \\
& F_{j+1}^{k} \equiv\left\lceil X_{j+1}^{k}\right\rceil
\end{aligned}
$$

Now check: If $F_{j+1}^{k}$ is in the set $\prod^{k}$, Increment $j$ by 1 and repeat the main operation

Else $\Pi^{k} \equiv \Pi^{k} \cup F_{j+1}^{k}$

If $n>N$

$$
\begin{aligned}
& X_{j+1}^{k}=\{\} \\
& \Pi^{k} \equiv \Pi^{k} \cup\left\lceil X_{j+1}^{k}\right\rceil
\end{aligned}
$$

\section{End}

\section{PERFORMANCE ANALYSIS}

Table.3. Simulation parameters for BER analysis

\begin{tabular}{|c|c|c|c|}
\hline Parameter & \multicolumn{3}{|c|}{ IDMA } \\
\hline & Fig.3 & Fig.4 & Fig.5 \\
\hline Information bits & 1024 & 1024 & 1024 \\
\hline Repetition code & $R_{r}=1 / 16$, & $R_{r}=1 / 16$, & $R_{r}=1 / 16$ \\
\hline Bit interleaver & Uniform random, TBI, TMI, logistic map \\
\hline Modulation & \multicolumn{3}{|c|}{ BPSK } \\
\hline Spread length & $N_{s}=16$ & $N_{s}=16$ & $N_{s}=16$ \\
\hline
\end{tabular}

\subsection{BIT-ERROR RATE ANALYSIS}

In this section, the quality of transmission of conventional IDMA is evaluated for proposed chaos based interleaver. Random Interleaver, tree based (TBI), logistic map interleaver and Tent map interleaver are considered for bit error rate (BER) analysis in conventional IDMA system. For simplicity, BPSK modulation is considered in multipath Rayleigh fading channel. The data length for each user is assumed 1024 bits and repetition code is used as a spreading code with the length $s l=16$. Simulation parameters are shown in Table. 3 for all simulation results.

In Fig. 3, simulation results show the comparative BER performance of logistic map based interleave with random interleaver. The data length is assumed 1024 bits, number of iterations are 10 and spread length $s l=16$ is considered. Simulation figure concludes that the performance of logistic map is nearly same as compared to RI and somewhat better for higher values of $E_{b} / N_{0}$. Hence, Logistic map algorithm can be more efficient for higher values of $E_{b} / N_{0}$.

In Fig.4, simulation results show the comparative BER analysis of one more chaos based Tent map interleaver with random interleaver. The data length is assumed 1024 bits, number of iterations are 10 and spread length $s l=16$ is considered. Simulation result verifies the performance of tent is better than random interleaver for higher values of $E_{b} / N_{0}$.

The comparative performance of logistic map and Tent map interleaver with conventional random interleaver is shown in Fig.5. The data length is considered to be 2096 bits, spread length $=16$ and iteration $=10$ is taken for performance analysis. Simulation results show that the Tent map based interleavers outperform the logistic map and random interleaver.

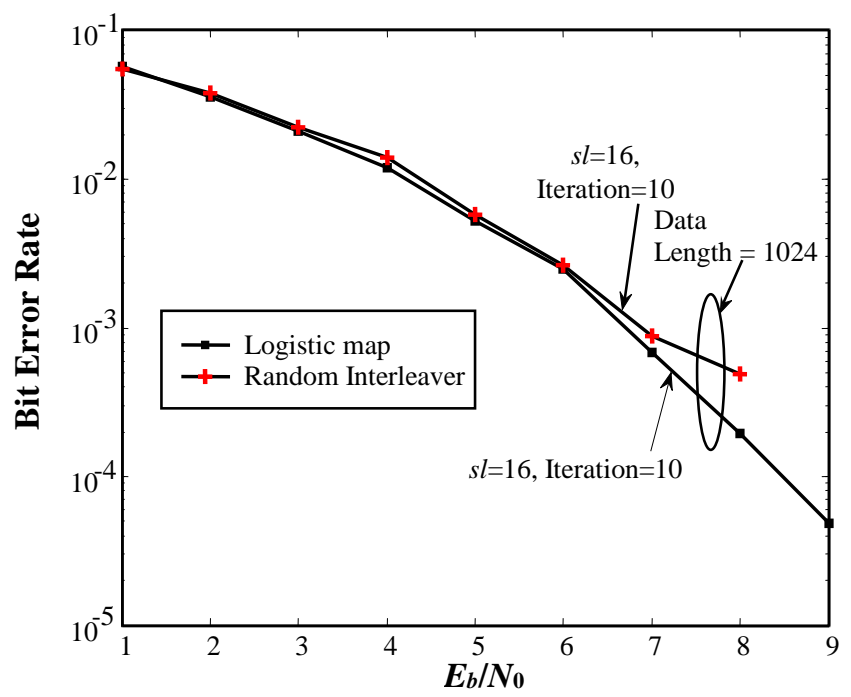

Fig.3. BER comparison of logistic map and random interleaver for data length $=1024$, spread length $=16$, iteration $=10$

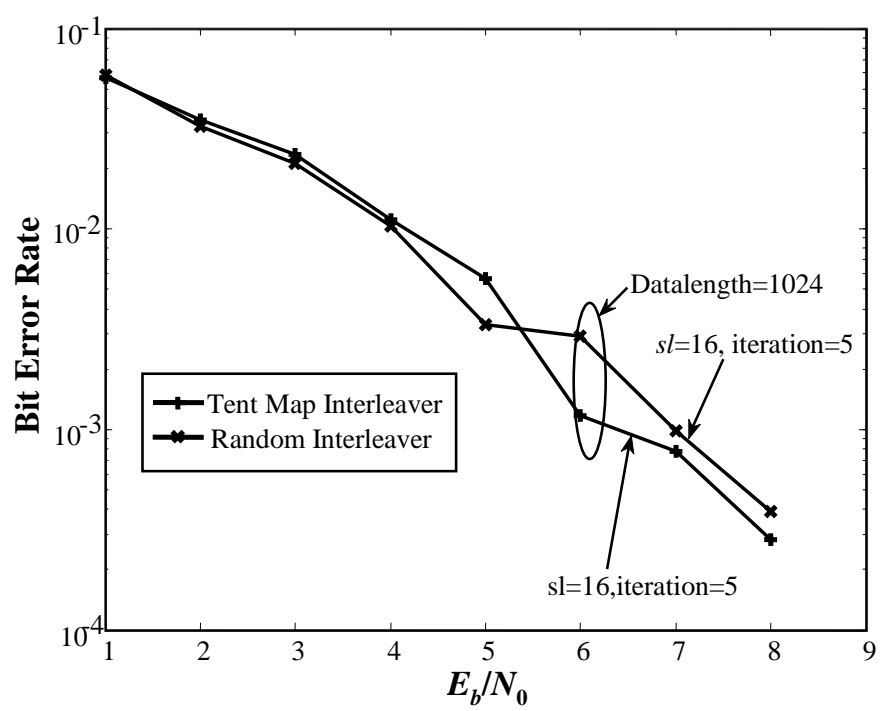

Fig.4. BER comparison of Tent map, random interleaver for data length $=1024$, spread length $=16$, iteration $=10$, Block $=5$ 


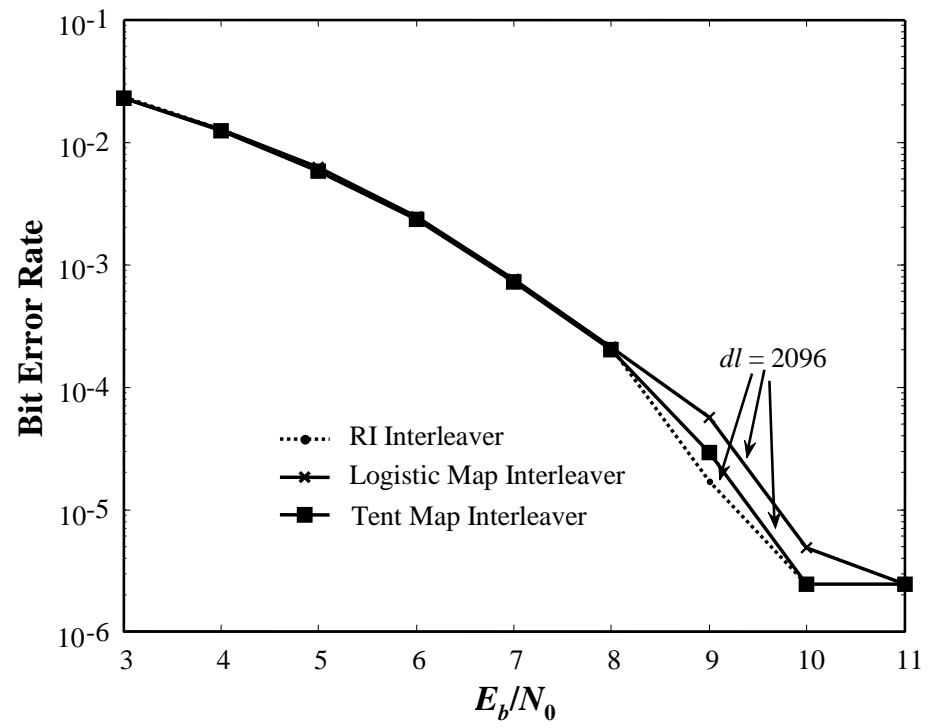

Fig.5. BER comparison of Tent map, random interleaver, and logistic map interleaver for data length $=1024$, spread length $=$ 16 , iteration $=10$, Block $=5$

\subsection{COMPUTATIONAL COMPLEXITY}

Other than BER analysis, Computational complexity is also an important parameter to decide the quality of communication. Here, complexity means that number of cycles required for the generation of spreading codes and interleaver matrix in terms of users. In this section, complexity is calculated for tent map interleaver and for other popular algorithms used for interleaver generation [5]. The Table.4 shows that the computational complexity increases with the number of user $k$ for the orthogonal, Nested and Tree based interleaver (TBI). It means complexity is dependent on the users for all mentioned schemes, except proposed design i.e. TMI. In fact, the complexity is $\mathrm{O}(1)$ which means that computational complexity of TMI is independent from the number of users.

Table.4. Number of cycles required for the generation of $k^{\text {th }}$ interleaver

\begin{tabular}{|c|c|c|c|c|}
\hline No. of users $(\boldsymbol{k})$ & OI & Nested Interleaver & TBI & TMI \\
\hline 1 & 1 & 1 & 1 & 1 \\
\hline 2 & 2 & 2 & 1 & 1 \\
\hline 4 & 4 & 4 & 2 & 1 \\
\hline 16 & 16 & 16 & 4 & 1 \\
\hline 50 & 50 & 50 & 5 & 1 \\
\hline
\end{tabular}

\subsection{MEMORY REQUIREMENT}

For the proper processing of signal at receiver, it is required that the interleaving matrix should be known to the receiver. The transmitter needs to transmit the interleaving matrix with the data. For the larger number of users, the large amount of memory is required to store interleaver matrix at the side of transmitter and receiver. It increases the memory requirement of the system. This issue becomes another challenge in the designing of interleaver for IDMA system. The proposed TENT map based interleaver algorithm suggests the need of only initialization parameter to generate a specific interleaver. Hence only single bit i.e. initialization parameter is required to share between transmitter (MS) and receiver (BS). So, the TMI is efficient in memory requirement. In Table.5, memory requirement for different interleaver is presented. The spreaded data is represented as chiplength $c l$ and $k$ is the number of users.

Table.5. Memory requirement (per user) for different interleavers

\begin{tabular}{|l|c|}
\hline \multicolumn{1}{|c|}{ Interleavers } & $\begin{array}{c}\text { Memory requirement } \\
\text { (chips/user) }\end{array}$ \\
\hline Random interleaver & $k \times c l \times \log _{2}(c l)$ \\
\hline Tree Based Interleaver & $2 \times c l \times \log _{2}(c l)$ \\
\hline Nested Interleaver & $c l \times \log _{2}(c l)$ \\
\hline Tent Map Interleaver & $c l \times \log _{2}(c l)$ \\
\hline
\end{tabular}

\section{CONCLUSIONS}

This paper proposes the chaos based algorithms for an efficient interleaver design. The interleaver design is based on the chaos theory and uses the logistic map and Tent map to generate interleavers for simultaneous users of pertinent system. The method achieves good bit error rate performance as well as it is potentially less complex computationally. The memory requirement is one of the major challenges in designing the interleavers but the simulation results verify that the storage requirement in chaos based interleavers is independent of users. Hence, a large number of interleaving sequences can be generated without investing additional resources. The research and findings also pave the path for further rectification and improvements in the method such as Tent map can be modified, so that IDMA based on TMI can be more suitable for secure communication.

\section{REFERENCES}

[1] Francisco J. Escribano, Alexandre Wagemakers and Miguel A. F. Sanjuan, "Chaos-based Turbo systems in Fading Channels", IEEE Transactions on Circuits and Systems, Vol. 61, No. 2, pp. 530-541, 2014.

[2] Akihiko Kitada and Yoshihito Ogasawara, "On a Property Specific to the Tent Map", Chaos, Solutions and Fractals, Vol. 29, No. 5, pp. 1256-1258, 2006.

[3] Li Ping, Lihai Liu, Keying $\mathrm{Wu}$ and W.K. Leung, "Interleave-Division Multiple-Access", IEEE Transactions on Wireless Communications, Vol. 5, No. 4, pp. 938-947, 2006.

[4] M. Eisencraft et.al., "Chaos based Communication Systems in Non-Ideal Channels", Communications in Nonlinear Science and Numerical Simulation, Vol. 17, No. 12, pp. 4707-4718, 2012.

[5] M. Eisencraft, D.M. Kato and L.H.A. Monteiro, "Spectral Properties of Chaotic Signals Generated by the Skew Tent Map", Signal Processing, Vol. 90, No. 1, pp. 385-390, 2010

[6] M. Shukla, V.K. Srivastava and S. Tiwari, "Analysis and Design of Optimum Interleaver for Iterative Receivers in IDMA Scheme", Wireless Communications and Mobile Computing, Vol. 9, pp. 1312-1317, 2009. 
[7] Ioachim Pupeza, Aleksandar Kavcic and Li Ping, "Efficient Generation of Interleaver for IDMA", Proceedings of IEEE Conference on Communications, pp. 1508-1513, 2006

[8] Shu-Ming Tseng, "IDMA based on Deterministic Interleavers", International Journal of Communications, Network and System Sciences, Vol. 3, No. 1, pp. 94-97. 2010.

[9] Zhang Chenghai and Hu Jianhao, "The Shifting Interleaver Design based on PN Sequence for IDMA Systems", Proceedings of International Conference on Future Generation Communication and Networking, pp. 1-6, 2007.

[10] Shan Liang, Qiang Hao, Li Jun and Wang Zhi-Quan, "Chaotic Optimization Algorithm based on Tent Map", Control and Decision, Vol. 20, No. 2, pp. 179-182, 2005.

[11] O.M. Kwon, J.H. Park and S.M. Lee, "Secure Communication based on Chaotic Synchronization Via Interval Time-Varying Delay Feedback Control", Nonlinear Dynamics, Vol. 63, No. 2, pp. 239-252, 2011.
[12] Sangeetha Manoharan and Vidhyacharan Bhaskar, "PN Codes Versus Chaotic Odes: Performance Comparison in a Gaussian Approximated Wideband CDMA System over Weibull Fading Channels", Journal of the Franklin Institute, Vol. 351, No. 6, pp. 3378-3404, 2014.

[13] Brahim Akbil, Guillaume Ferre and Driss Aboutajdine, "The NLM Interleaver Design for IDMA System", Proceedings of International Conference on Complex Systems, pp. 1-6, 2012.

[14] Brahim Akbil and Driss Aboutajdine, "Improved IDMA for Multiple Accesses of 5G", International Journal of Communication Networks and Information Security, Vol. 7, No.3, pp. 138-146, 2015.

[15] Aasheesh Shukla and Vinay Kumar Deolia, "Performance Analysis of Chaos based Interleaver in IDMA system", ICTACT Journal on Communication Technology, Vol. 7, No. 4, pp. 1397-1401, 2016. 